\title{
THE CENTRIFUGAL PATHWAY FOR MICTURITION WITHIN THE SPINAL CORD
}

\author{
BY \\ P. W. NATHAN and MARION C. SMITH \\ From the National Hospital for Nervous Diseases, Queen Square, London
}

In previous papers we have described the location in man of the spinal pathways subserving sensation from the lower bowel and the motor aspects of defaecation (Nathan and Smith, 1953), and the spinal pathways subserving sensation from the bladder and urethra (Nathan and Smith, 1951; Nathan, 1952). It remains now to show the location in the spinal cord of the pathways used in the conscious control of micturition and those used for the coordination of bladder functioning.

\section{Historical}

The location of these fibres in the spinal cord both of man and other animals has been relatively neglected. Most observers who have studied the functional anatomy of micturition have considered the peripheral nerves of the bladder, the effects on the bladder of total transverse lesions of the spinal cord, and they have studied those centres in the brain, stimulation or lesions of which affect the tone and other reflex activity of the bladder. The first worker to investigate the location of the tracts subserving micturition was Budge; he worked on the subject in 1842 and published his results and conclusions in 1864. He obtained bladder contractions in the dog, cat, and rabbit by electrically stimulating the cerebral peduncles, the inferior cerebellar peduncles, and the region between these peduncles and the calamus scriptorius. In one animal, he cut through the posterior columns, and obtained contraction of the bladder, rectum, and Fallopian tubes from stimulating the rest of the spinal cord; he concluded that the descending pathway must lie in the anterior half of the cord. Mosso and Pellacani (1882) criticized Budge's work, as they found that his mode of stimulating the cord was bound to cause much spread of the current. They induced bladder contractions in the dog by painfully compressing the paws. To determine the spinal course of the tract conveying impulses giving rise to these contractions, they carried out various partial sections of the cord. Their conclusions were: "Dans les cordons antérieures de la moelle et dans la partie antérieure des cordons latéraux, il ne passe pas de fibres motrices de la vessie." Stewart (1899), who worked on the cat, found that " bladder contractions can be produced by stimulating the cord at any point"; and he also found that "stimulation of the cord often causes an already contracted bladder to relax". He came to the conclusion that, "Above the vesical centre ... impulses pass down the cord in the posterior half of the lateral columns only. . . Below the level of the centre the impulses were shown to pass in the lateral columns." He placed the vesical centre in the lumbar cord. Spiegel and MacPherson (1925) found that in the cat complete section of the anterior and posterior columns failed to interrupt the pathway; and they concluded, by increasing the area of cord sectioned, that the fibres subserving bladder contraction must run in the lateral columns. Further work on this subject in the cat led Hunsicker and Spiegel (1933) to conclude that the descending corticofugal tracts to the bladder are conveyed both by pyramidal and extrapyramidal pathways.

In our opinion, the best work on the location of the tracts subserving micturition is that of Barrington (1933). He summed up the results of his work, in 1948, as follows:

"In the cat, micturition is affected in the same way as by a spinal transection if the periphery of the dorsal half of the lateral column is destroyed on both sides in the upper lumbar region, but it remains unaffected if symmetrical lesions are made in other parts of the cord at this level. It is probable that the periphery of the dorsal half of the lateral columns is the part of the cord containing the tracts used in micturition in the segments above the lumbar region."

He also showed (1933) that:

"The centrifugal and the centripetal paths of micturition undergo extensive crossing in the segments from which the pelvic nerves arise and possibly in the segment next in front of these."

Wang and Ranson (1939) caused bladder contractions in the cat by stimulating the hypothalamus, and sought for the descending pathway conveying impulses giving rise to the bladder contractions by making various lesions; their most caudal lesion was 
at the first and second cervical segments. They came to the conclusion that the descending tract mediating impulses to the bladder lies at this level in the antero-lateral column. Levin and Langworthy (1937) showed that lesions in the cat's tegmentum, not involving the cerebral peduncles and thus sparing the cortico-spinal tracts, cause increased responses of the detrusor musculature to stretch and to "peripheral stimulation in the sensory domain of the sacral and coccygeal segments", changes which eventually lead to a decreased vesical capacity. Later, Langworthy (1940) showed that electrical stimulation of the cortico-spinal tract causes bladder contraction. Thus, Langworthy has brought forward evidence that there are pathways both within and without the cortico-spinal tract in the cat which are concerned with bladder functioning. Tower (1940), however, found in the monkey that dividing the cortico-spinal tract bilaterally caused "no urinary or faecal incontinence or retention".

Thus, the evidence for the location of the descending pathways to the bladder centre in the cat is fairly consistent: the pathways lie in the lateral column of the cord. It is not agreed whether the fibres lie in the dorsal or ventral parts of these columns. The evidence indicates that some of the fibres run with the cortico-spinal tract and some run outside this tract.

Before the turn of this century, no mention of the location of these tracts within the human spinal cord had been made. In 1900, von Czyhlarz and Marburg wrote that the tracts connecting the cerebrum with the sacral cord "seem to run in the neighbourhood of the pyramids. But in order to get permanent disorder of bladder function, it seems that both pyramids must be damaged, and damaged to a large extent". Nineteen years later, Müller (1918) still had to state:

"We have no information concerning the paths in the spinal cord by which the bladder centres receive their stimulation or inhibition from the brain. We do not know in what part of a section of the spinal cord we should seek them. We have no proof that the impulses to the smooth muscles of the bladder make use of the centrifugal pyramidal tract."

And in 1926, Dennig wrote that doubt might be cast on the hypothesis that the motor tract in man lies in a similar position to that which it occupies in animals. He wrote that lesions of the spinal cord giving rise to severe disorders of micturition may not involve the lateral cortico-spinal tracts, and that lesions of the lateral cortico-spinal tract may cause no disorders of micturition. He pointed out, however, that it is hazardous to draw conclusions from clinical conditions, for the upper motor neurone lesion might not involve the nerve supply to all muscle groups.

The first workers to present any experimental work on man designed to show the location of these tracts were Foerster and Gagel (1932). They stated in their text that bilateral cordotomy " repeatedly" gave rise to retention of urine, although the retention was always temporary. They drew the conclusion that :

" the descending tract in the spinal cord subserving bladder innervation runs partly in the antero-lateral column. This conclusion has to be drawn from the retention of urine that initially follows the operation. But vesical tracts must also run in other columns, for even after the most complete bilateral anterolateral cordotomies, the bladder behaviour shows complete recovery."

It must be pointed out, however, that this part of their study of the effects of cordotomy is inadequate, and that the very short case histories appended to their text do not always support the conclusions they drew. In their paper there are 17 cases of bilateral cordotomy; in two of these cases the state of micturition of the bladder is not mentioned. In a further six cases the evidence presented does not give support to the statements they made. In one case, due to collapse of the vertebrae, there $\frac{}{\oplus}$ was retention of urine before the cordotomy; and 0 in another case the collapse of the vertebrae occurred one week after the cordotomy, making further 8 observations on the effects of cordotomy impossible; another patient died one week after the operation. Two patients died three months and eight months respectively after the cordotomy, from cystopyelitis -a condition which usually follows paralysis of the bladder. A further patient two months after the cordotomy still had retention of urine.

Langworthy and Lewis (1935) concluded from two cases of disseminated sclerosis which they studied in detail (unfortunately without obtaining postmortem evidence), that their patients' urgency and frequency of micturition was related to " damage to the lateral columns of the spinal cord in the neighbourhood of the cortico-spinal tracts ". In 1940, Langworthy, Kolb, and Lewis stated that the pathway " controlling tone in the vesical muscle . . . probably lies in the lateral columns close to the cortico-spinal tracts". Presumably, they drew this conclusion from the two cases already mentioned and the evidence from experimental work on animals.

Wruck (1943) stated that extensive antero-lateral cordotomies do not cause bladder disturbance; and from this he concluded that the centrifugal tracts to the bladder probably do not lie between the anterolateral and postero-lateral columns. No evidence was given in his paper apart from the bald state- 
ment; it seems that his views were based on Foerster's experience, already quoted.

Thus we see that in man there is no adequate evidence apart from that provided by Foerster and Gagel; and this is most unsatisfactory. Langworthy et al. wrote in 1940:

"Pathological studies in man are needed to delimit more clearly the pathways controlling micturition. It will be of interest to observe whether these studies will confirm the anatomical localization suggested by animal experimentation."

Such studies in man are presented here.

\section{Effects on Bladder Functioning of Dividing the Spinal Cord}

When all the spinal tracts subserving micturition have been divided, the resulting disturbance of function resembles, though it is not identical with, that which follows when the spinal cord has been totally divided. Before considering the present series of cases, the functioning of the bladder when the cord is divided will be briefly reviewed. It is immaterial at what level such a transection occurs provided it divides the sacral centre from those centres above the medulla oblongata that play a part in the control and coordination of micturition.

Transection of the cord interrupts the sensation of pain from the bladder and the normal sensation underlying the desire to micturate, and the control of micturition permanently; but the behaviour of the automatic bladder, cut off from impulses arising above the spinal cord, is a changing one. This changing course of reflex and postural activity is usually considered to pass through three stages; these will now be briefly considered.

Most writers on this subject write of the state of the visceral musculature of the bladder as being regulated by the same factors that regulate the state of the somatic musculature. This is an assumption. Whether this assumption is justified will not be considered here, as it does not influence the observed behaviour of the bladder.

The bladder, like all muscle, has tone or posture. Posture was designated by Sherrington (1915) as:

" that property of the bladder by virtue of which it solves the problem of acting as a reservoir for quantities of fluid of very varying volume from one occasion to another without allowing the intravesical pressure to attain the reflex stimulus threshold height with one particular fluid quantity only."

This postural activity is under the constant influence of centres situated cranial to the spinal cord.

When the spinal cord is suddenly divided, the bladder is then, according to most observers, in a state of flaccidity or atonicity. The cystometrograph shows the flat type of curve typical of low pressure. In fact, the viscus certainly possesses some intrinsic tone, for it is not completely flaccid even when all 3 the nerves between it and the sacral centres have been divided. Further, some recent workers consider that the type of curve seen in the early stage after division of the cord results from stretching the musculature of a bladder in which the micturition reflex is absent. At this time, there is no vesical reflex activity; there are no detrusor contractions and no micturition reflex, and these cannot be induced by the injection of carbaminoyl choline. The sphincters are tightly shut. Just as with the somatic musculature, in which the stage of flaccidity with spinal shock eventually gives way to a stage of increased reflex activity characteristic of the spinal cord functioning independently of the centres cranial to the medulla oblongata, so in the bladder there follows a stage of increased reflex activity; reflexes are increased in the sense that they fire off with lesser stimuli and that the resultant contraction is larger and longer lasting. There is spasticity-an increased resistance to passive movement. In the bladder, this can be made apparent by filling the viscus so as to stretch the musculature, while the pressure is recorded. This spasticity is thought to be due to increased reflex activity, the result of heightened stretch reflexes. Cystometrography at this time shows a more active detrusor musculature; the tone is increased, the pressure curve rises very steeply compared with the normal, and there are sharp waves of increased pressure. When these sharp waves occur, the sphincter mechanism of the bladder and urethra relaxes. Periodic micturition now occurs; and the urine can be manually expressed with relative ease. Thus, retention of urine with decreased reflex activity changes to periodic automatic micturition. This periodic micturition can be so efficient that the bladder may expel all its contents. Eventually, posture, as designated by Sherrington above, becomes conspicuously absent; for much spontaneous change in pressure occurs with a constant volume of fluid. The pressure is constantly high with a small volume of fluid; for instance, $75 \mathrm{ml}$. of fluid may be held at a pressure of $100 \mathrm{~cm}$. of water. The reaction of the bladder musculature is now greatly enhanced. Sudden contractions of the musculature often expel the catheter as well as the urine. Anything causing the slightest increase in tension on the bladder musculature, such as a rise in intra-abdominal pressure, or a slight movement, gives rise to the micturition reflex-contraction of the detrusor with opening of the sphincters and expulsion of urine; but this reflex is inadequately sustained, so that although small amounts of urine are spurted out, large amounts often remain in the bladder. When the pressure of the bladder is very high, these reflex expulsions of urine may lead to the wrong conclusion that urine is dribbling away 
continuously, as with a patulous sphincter. Cystometrography at this time shows a steeply rising curve in nearly all cases; however, in a few cases the curve remains flat once a certain high level has been reached.

The normal coordination between the detrusor musculature and the sphincter musculature is severely disturbed. Denny-Brown and Robertson (1933) have suggested that this is partly due to the fact that intercurrent stimuli from the lower part of the body cause a sudden reflex shutting of the sphincter with consequent cessation of micturition before the bladder has been emptied.

The progress through these recognized three stages is gradual. Stage $I$ is the stage of absence of detrusor contractions and of the micturition reflex; Stage II is the stage of spasticity with a steeply rising cystometrographic curve; and Stage III shows the features of Stage II in more pronounced form; there is intense spasticity, vigorous contractions of the detrusor being evoked by small quantities of urine and by intercurrent stimulation of the more caudal parts of the body. This manner of considering the progress of bladder functioning as passing through three stages will be followed here, as it allows our work to be compared with previous work, and as it permits more concise presentation of our results.

\section{Effects on Bladder Functioning of Cutting Spinal Pathways Subserving Micturition}

It was said above: “ When all the tracts subserving micturition have been divided, the resulting disturbance of function resembles that which occurs when the spinal cord has been totally divided." The slight differences in bladder functioning when the cord has been divided and when the spinal tracts specifically subserving micturition have been divided will now be considered.

(a) Sensation.-Patients with division of the tract conveying impulses subserving the normal sensation of the desire to micturate have nevertheless various other ways of finding out when the bladder is full and when it is going to evacuate its contents automatically. This aspect of the subject has been investigated and discussed by Nathan and Smith (1951) and by Nathan (1956 $a$ and $b$ ).

(b) Conscious Control.-The ability to control consciously the levatores ani, and in particular their anterior parts, the pubo-coccygeus muscles, and the external sphincter is lost. How far this loss interferes with the ability to start micturition is not really known. In normal micturition the neck of the bladder descends in the pelvis and the anterior parts of the levatores ani contract and the detrusor contracts at about the same time; Nesbit and
Lapides (1948) have shown that bilateral pudendal nerve block does not interfere with the ability to start micturition. With regard to stopping micturition, the external sphincter is important, and they showed that bilateral pudendal nerve block does interfere with this mechanism. It seems that one may state that without control of the perineal musculature the patient cannot start or stop micturition in a normal manner. However, such a patient when micturition has reached Stages II and III, may be able to empty his bladder, at least partially, by inducing a reflex contraction of the detrusor. Various ways of doing this are found by patients; they may press on the abdomen, hold the breath and strain, or they may flex the thighs and trunk. Although such a patient cannot actively stop micturition, it can often happen that micturition continues only as long as straining is sustained, and in such a case the patient can stop micturition merely by ceasing to strain. The normal inhibition of the rising pressure exerted by the bladder musculature, which is a feature of bladder control, can in no way be substituted when the relevant tract is divided. It is important to realize that the paralysis of the muscles of the pelvic floor and of the perineum can occur without any concomitant paralysis of the lower limbs, and with some neurological lesions one can find severe paralysis of the lower limbs with minimal involvement of the movements of the musculature of the pelvis and perineum.

(c) Postural and Reflex Activities.-There is noo difference in these activities in those cases in which the cord is totally divided and in those in which only the relevant tracts in the cord are divided.

Such are the features of the functioning of the bladder to be found when the tracts within the spinal cord subserving micturition are divided. They can be made manifest in an acute experiment-observing the posture or tone of the bladder during the operation of dividing the relevant pathways in the cord, and in a chronic experiment-day-to-day observations of the postural and reflex activities after these tracts have been divided. Observations will not be made here on the sensory aspect of the subject as it has been considered in previous papers.

\section{Methods of Investigation}

Both the clinical and the histological methods of investigation employed were described in our previous paper on the afferent pathway in the cord (Nathan and Smith, 1951).

\section{Material}

Out of a series of 60 cases of cordotomy performed for the relief of pain in which full histological examination of the spinal cord and brain has been 
carried out, 21 cases of bilateral cordotomy and 15 cases of unilateral cordotomy were studied in detail with regard to the functioning of the bladder and micturition; one case of thrombosis of the posterior inferior cerebellar artery was also investigated, although no post-mortem examination was made, the patient still living. The evidence from the other cases of this series supports the conclusions drawn from this selected material; but as these patients were not investigated in such detail, no further mention of them will be made.

Although we now have a large number of cases, it is surprisingly difficult to collect a sufficient number of suitable cases for such a study as this. All the patients, except the one with a thrombosis of the posterior inferior cerebellar artery, had cancer. Patients in whom the cancer involved the central nervous system were excluded from the series; but those in whom only the peripheral nerves were involved had to be used. For in most patients needing cordotomy for the relief of pain the peripheral nerves are involved by the tumour. In some of these patients there was involvement of the sacral nerves on one side. It might be thought that as there was a lesion of the sacral nerves, the effects of dividing the pathway in the spinal cord could not be properly estimated; and it could further be objected that comparisons between cases cannot properly be made when some of the patients have the sacral nerves involved by growth and others have the peripheral nerve supply intact. And in addition to these objections, it might be considered that as the lesions made in the spinal cord vary considerably from case to case, the number of factors playing a role is so large that one case can hardly be compared with another. Nevertheless, it seems to us that the inclusion of patients with involvement of the sacral nerves on one side is justified. There is in this series a sufficient number of cases without any involvement of the sacral nerves, and so it is quite clear what the results of dividing the tracts in the cord are. A further reason is that each patient was investigated before and after the cordotomy; and so, whatever sort of bladder function was found before this operation, any sudden changes in it could be attributed to the effects of dividing the tracts in the
Fig. 1.-Cystometrogram made during the cordotomy. At each arrow an incision was made into the antero-lateral part of the spinal cord. spinal cord. And finally, the kind of bladder function that results from a lesion of the peripheral nerve supply is different from that kind which results from a lesion of the spinal tracts.

The Immediate Effects of Dividing the Tracts

An acute experiment demonstrating the loss of tone caused by sudden division of the lateral and antero-lateral parts of the cord is shown in Fig. 1 (Case 47). Cystometrography was performed during the course of the bilateral cordotomy; the anaesthesia was kept as light as possible to avoid any effects of the anaesthetic on the centres coordinating micturition. The pressure in the bladder was rising rather rapidly before the incision was made; this may have been due to the inevitable interference with the spinal cord. When one side of the cord was incised, the bladder pressure fell from $36 \mathrm{~cm}$. to $18 \mathrm{~cm}$. of water; when the second side of the cord

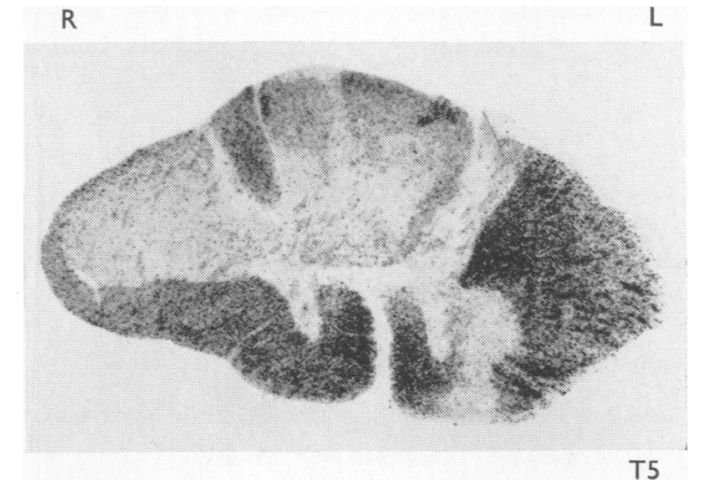

Fig. 2.-Case 47: Transverse section through lesion (Marchi preparation). 


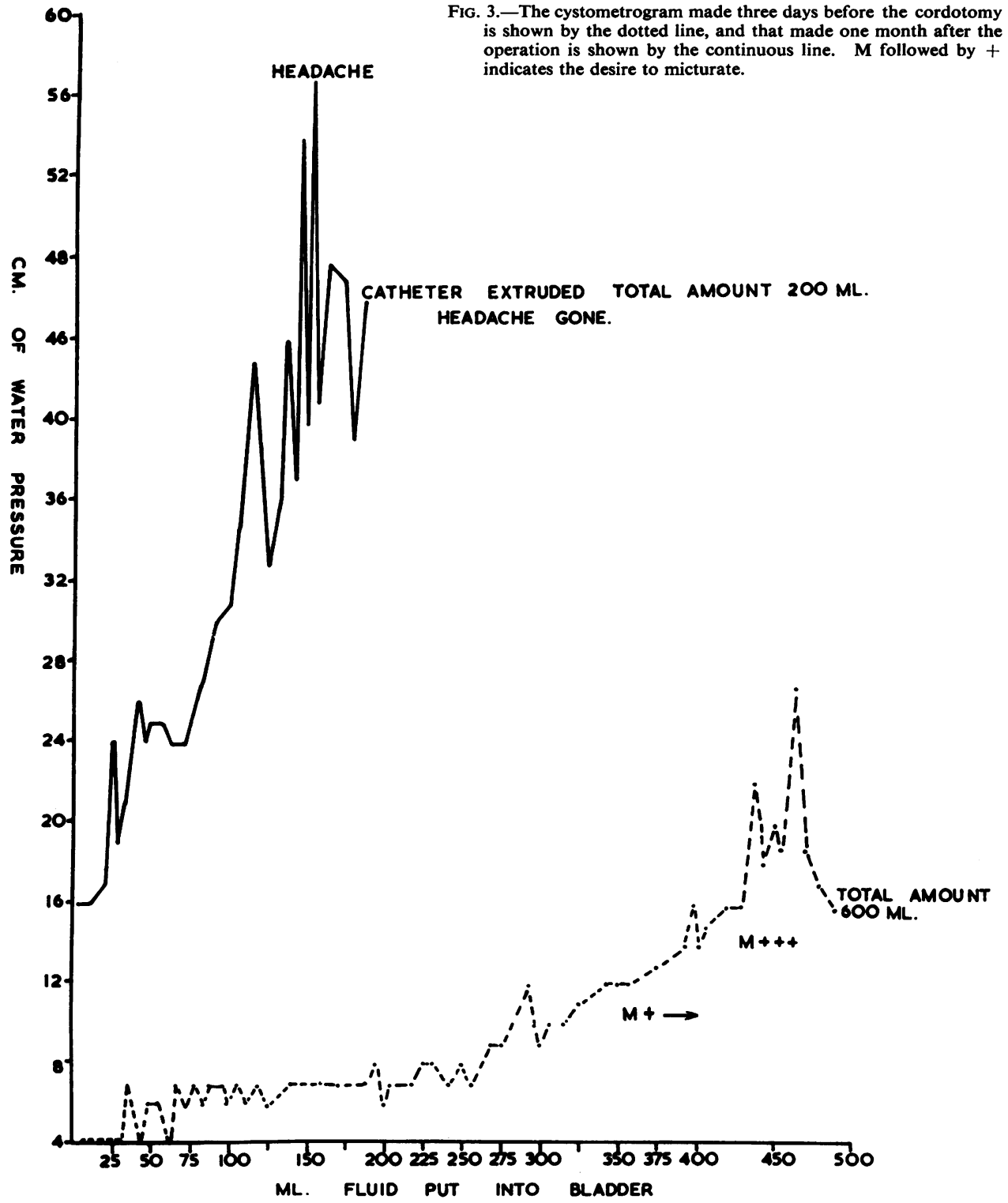

was incised, the bladder pressure fell a further $5 \mathrm{~cm}$., and it remained at this level for the rest of the time of the operation, in spite of filling of the viscus.

The extent and location of the lesions made in this spinal cord, in the thoracic segment, can be seen in Fig. 2.

\section{Chronic Effects of Dividing the Tracts Bilaterally}

Two cases will be described in which there was good evidence of bilateral division of all descending tracts.
Case 1.-Mrs. M. had a bilateral cordotomy for the pain due to carcinoma of the cervix uteri. Following the operation there was analgesia throughout the right side of the body below the sixth thoracic dermatome and throughout the left side of the body below the ninth thoracic dermatome. The function of the bladder was that of Stage I; and three weeks after the operation $0.25 \mathrm{mg}$. of carbaminoyl choline chloride had no effect on the bladder. One month after the operation Stage II was reached. Fig. 3 shows the cystometrogram performed before the cordotomy, and that performed after the cordotomy during Stage II. Comparing the two, the 
$\mathbf{R}$

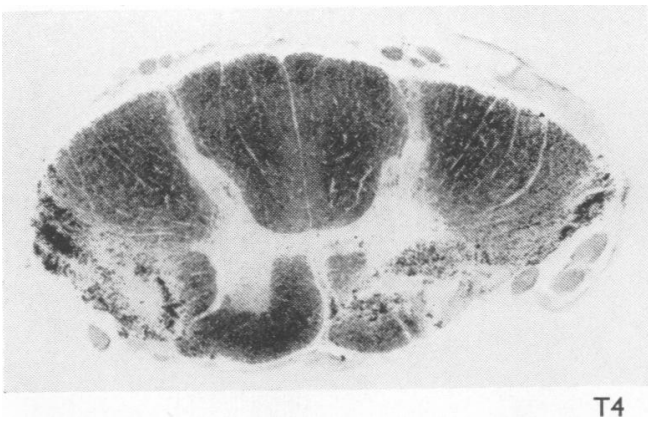

FIG. 4.-Case 1: Transverse section through lesion (Marchi preparation).

pressure on starting, when only enough fluid is in the system to take a reading, was $4 \mathrm{~cm}$. before the operation, and 16 to $18 \mathrm{~cm}$. after it. Before the cordotomy the pressure remained under $12 \mathrm{~cm}$. as long as the contents were below $375 \mathrm{ml}$.; then, with the increasing desire to micturate, the pressure began to rise steeply. Strong detrusor contractions started when the bladder contained $500 \mathrm{ml}$. In Stage II, the curve showed no horizontal component; it rose steeply as the bladder was filled, and there were strong detrusor contractions throughout. Finally, when only $200 \mathrm{ml}$. was in the bladder, there was such a strong contraction that the catheter was extruded. Before the operation, as the bladder was filled the patient was making a conscious effort to hold urine; after the operation, she felt she could not influence the bladder in any way. At necropsy 68 days after the operation, the bladder was seen to be trabeculated.

The location and extent of the bilateral lesions in this case are shown in Fig. 4.

Case 7.- Mrs. $Y$ had a bilateral cordotomy for the pain due to a colloid carcinoma of the cervix uteri. Following the operation there was analgesia throughout the right side of the body below the tenth thoracic dermatome and throughout the left below the eighth thoracic dermatome. The function of the bladder was that of Stage I; Stage II was reached 12 days after the operation.

The location and extent of the bilateral lesions in this case are shown in Fig. 5.

From these three cases-the two described immediately above and the one given to show the acute effects of dividing the tracts-deductions can be drawn on the location of the fibres used in the control and coordination of micturition. These cases were typical of the group; 16 of the 21 studied showed this complete syndrome. The smallest area of degenerating fibres common to the six sides of the three cords presented must contain the majority of the centrifugal fibres concerned in micturition. It can be seen from Figs. 2, 4, and 5 that this area lies on the equatorial plane of the cord. In all the cases in which there was the complete syndrome,
R
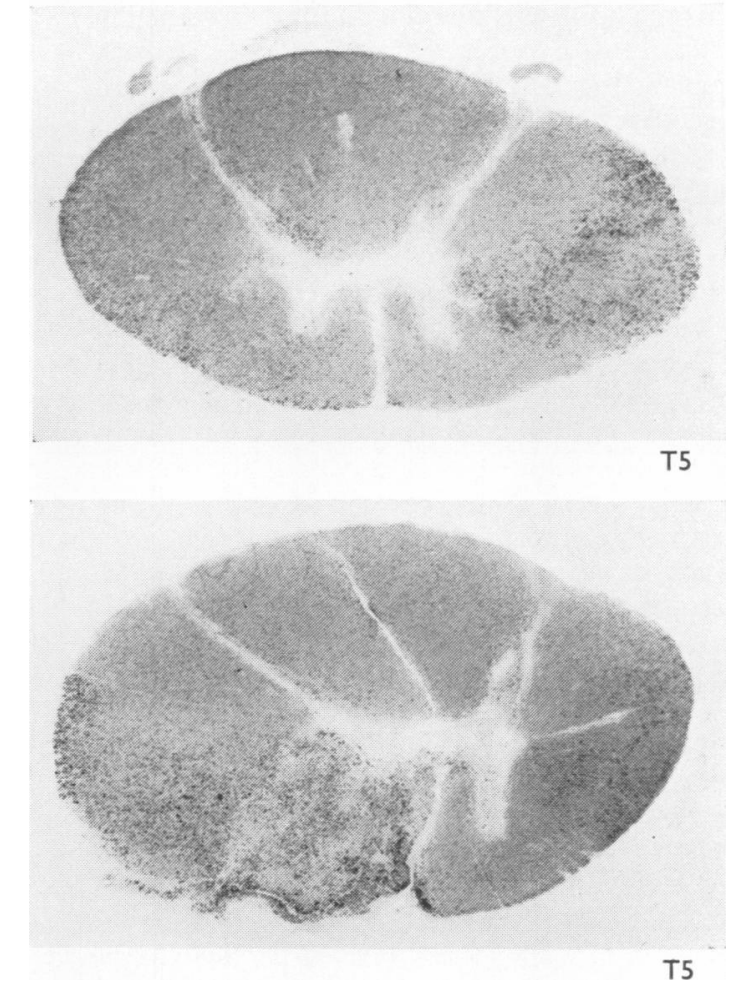

Fig. 5.-Case 7: Transverse section through lesion (Marchi preparation).

the lesions involved this region of the cord. In five other cases the typical syndrome was present for weeks, and then there was more recovery from this state than is usually found with complete lesions. It was interesting to note that in these cases the lesions made at operation did not involve this region as completely on both sides of the cord as it did in those cases with the complete clinical syndrome.

We thus conclude that the majority of the centri-

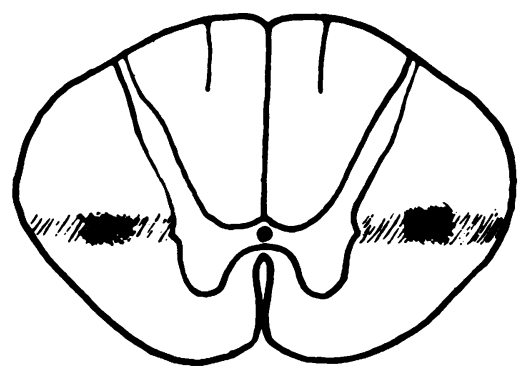

Fig. 6. -The region within the spinal cord of the centrifugal pathway for micturition. The majority of the fibres probably lie within the region indicated by cross-hatching. 
fugal fibres descending from the brain must lie in this region of the lateral columns shown in Fig. 6.

In most of our cases, and in those presented so far, the lesions were made in the thoracic cord. In one case the incisions were made at the fifth and sixth cervical segments, and in two cases at the first and second lumbar segments. The location of the lesions in these three cases showed that the efferent pathway lies in the same region of the lateral columns in these parts of the cervical and lumbar cord as it does in the thoracic cord.

More evidence that the pathways lie in this part of the cord can be obtained from those patients who did not have the syndrome of complete division of the tracts but showed a part of the syndrome or more recovery from the stages of the syndrome than is possible with total division of the relevant tracts. Further evidence is also gathered from those patients who had the incisions made in the neighbourhood of these tracts, and yet had no disturbance of bladder function.

\section{Chronic Effects of Partial Division of the Tracts Bilaterally}

The following case shows that bilateral lesions anterior to the region shown in Fig. 6 do not cause the complete syndrome.

Case 21.-Mr. M. had a bilateral cordotomy for the pain due to a chondrosarcoma of the ilium. Although the operation was bilateral, the incision on the left side of the cord was made purposely superficial and somewhat anterior. Following operation there was complete analgesia throughout the left side of the body below the ninth thoracic dermatome, and hypoalgesia on the right side of the body below the ninth thoracic dermatome. For the first week after the cordotomy there was complete retention of urine. By the end of the first fortnight, however, except for some frequency of micturition, bladder functioning was normal. He could micturate without straining and he could stop micturating in the middle of the act; he could hold urine for half an hour or more after feeling some desire to micturate. This state continued for five months. After this time, it was seen through the cystoscope that the base of the bladder was involved in the spreading tumour; and so further conclusions on bladder functioning could not be attributed solely to the lesions in the spinal cord. The lesions in the cord of this case are shown in Fig. 7. It is probable that on the left side some of the relevant fibres have been cut and that on the right side they have not been involved.

\section{No Effects after Bilateral Lesions in Neighbourhood of the Tracts}

The following case shows that bilateral lesions posterior to the region shown in Fig. 6 do not cause the syndrome.

Case 22.-Although the operation was bilateral, the incision on the left side of the cord did not involve any

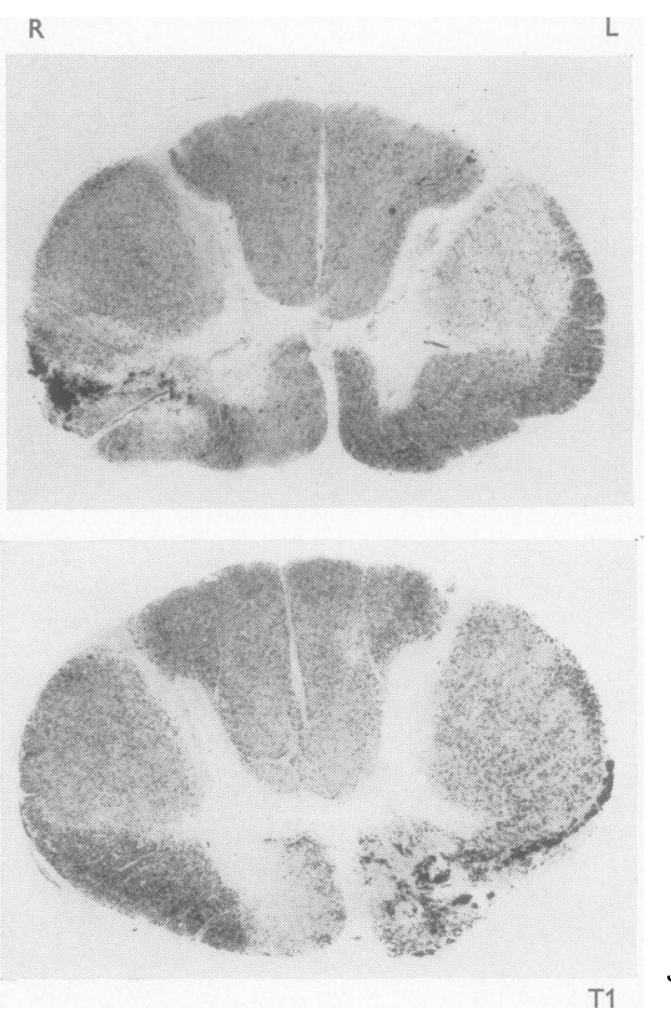

FIG. 7.-Case 21: Transverse section through lesion (Marchi preparation).

sensory fibres. Following operation, the only part of the body where pain sensibility was diminished was on the left in the first and second sacral dermatomes; it was slightly diminished in the third sacral dermatome. The

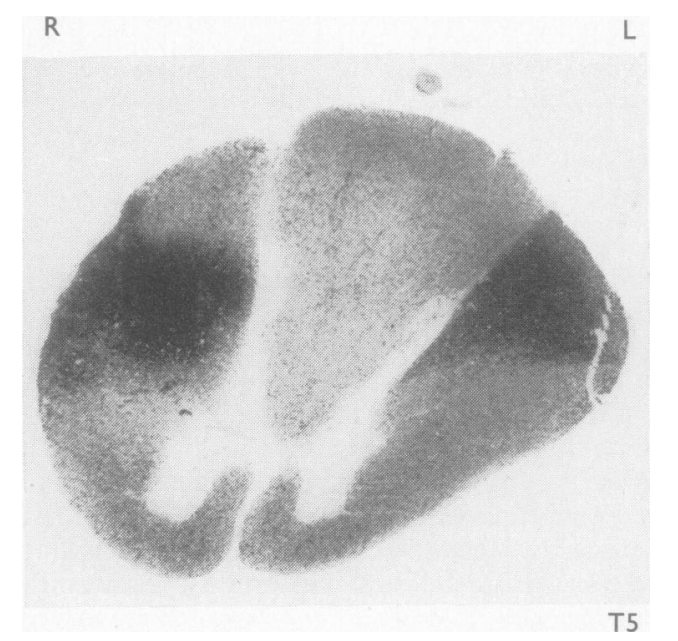

FIG. 8.-Case 22: Transverse section through lesion (Marchi preparation). 
R

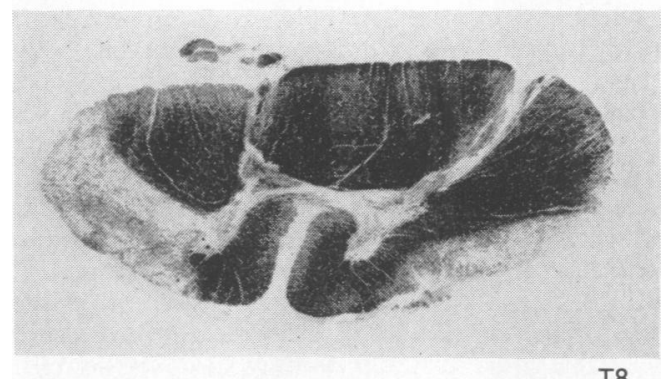

Fig. 9.-Case 11: Transverse section through lesion (Weigert Pal preparation).

patient developed no disturbance of micturition of any sort; he never had to be catheterized, he could start and stop micturition when he wanted, and he passed a normal amount of urine at normal intervals of time. The lesions in the cord are shown in Fig. 8 . It will be seen that the equatorial plane is not involved, with the possible exception of a few fibres on the right side.

Thus Cases 21 and 22 serve to show the boundaries of this region where the centrifugal fibres are thought to run. In Case 21 the lesion on the right side is at the anterior limit of the region; and in Case 22 both lesions probably lie just posterior to it. Other cases with lesions not involving this region of the spinal cord showed no disturbance of micturition or its conscious control.

In a previous paper (Nathan and Smith, 1951) on the afferent pathway from the bladder, we showed that the afferent pathway lies in the same region as that which we are now proposing for the efferent pathway. In one patient, Case 11, there was clinical evidence of bilateral division of the afferent tract with only partial involvement of the efferent pathway. The lesions in the cord, shown in Fig. 9, involved the lateral part of the region shown in Fig. 6. Thus, this one case may be regarded as evidence suggesting that the centripetal pathway lies lateral to the centrifugal pathway.

We have now shown where the tracts lie in the cervical, thoracic, and lumbar regions of the cord. It remains to consider their position in the sacral region. Fig. 10 shows the distribution of the degenerating fibres in the lower lumbar and the sacral segments, following a unilateral cordotomy in the third cervical segment. Degenerating fibres are seen in the lateral column of the lumbar section and considerably fewer fibres are to be seen in the same region of the sacral segments. When a comparison is made between this case and other cases with lesions involving slightly different parts of the lateral columns, it is seen that the more anterior of the degenerating fibres shown in Fig. 10 are those concerned with the conscious control and coordination of bladder functioning.

Degeneration in fibres to the sacral micturition centre is accompanied by degeneration in descending fibres to the neurones for the lower limbs. But as the neurones for the limbs are situated mainly cranial to the third sacral segment, there are, among those degenerating fibres still present at, and caudal to this segment, the fibres concerned with micturition, defaecation, and the pelvic musculature. In our series of cases only a few fibres are to be seen at these levels; and even in the cases in which the cordotomies were performed in the lumbar region more fibres are not seen. This reduction in the
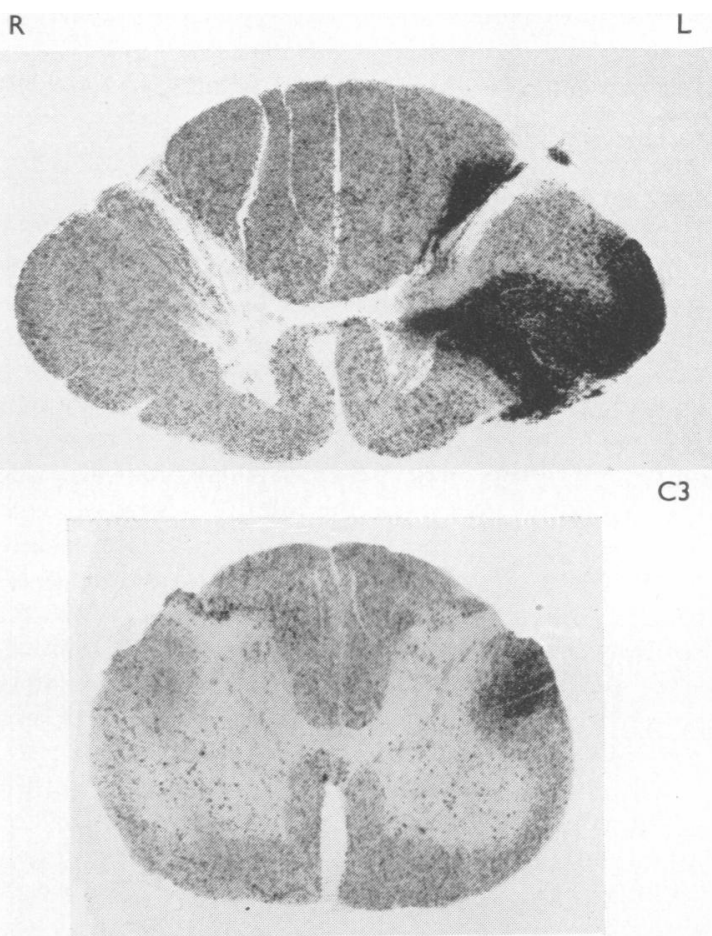

L3

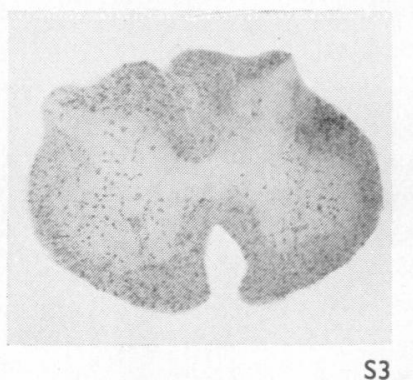

FIG. 10.-Case 37: Transverse section through lesion in cervical segment, and sections showing resultant degeneration in lumbar and sacral segments (Marchi preparation). 
number of fibres between the lumbar and sacral regions may seem surprising. The reasons for the small number of degenerating fibres are probably the following. As is well known, the degeneration of the terminal parts of the axon is completed much sooner than that throughout the rest of the axon, and so the terminal parts cannot always be seen in those cases where degeneration can be traced throughout the rest of the tract. Also, it could be that a few of the centrifugal fibres relay in the grey matter of the cord between cervical or thoracic segments and the sacral segments; in this case, there would be no degeneration in the axons having their cells of origin caudal to the axons divided in the cordotomy lesion.

\section{Chronic Effects of Division of Tracts Unilaterally}

The clinical picture resulting from division of the tracts on one side of the cord can now be given; for in certain cases it can be shown histologically that the relevant tracts have certainly been divided.

All patients who had the pathway subserving control of micturition divided unilaterally had no disturbance of voluntary control. Thus, one may conclude that a tract on one side of the cord is enough to ensure the normal function of starting and stopping micturition. (Needless to say, this statement is true only if the peripheral pathway and the structures, such as the bladder, urethra, and levatores ani, are themselves free from abnormalities.) Normal voluntary control is not necessarily present for the first four or five days after the cordotomy; for during this time (and immediately after any operation on the spinal cord) there may be retention of urine. But after a few days normal control is regained.

All patients who had the pathway subserving the postural and reflex activities of the bladder divided unilaterally showed an alteration in these functions. Although the patients were themselves unaware of any abnormal functioning of the bladder, an abnormality could be demonstrated by comparing the pre- and the post-operation cystometrograms: in all these patients the post-operation differed from the pre-operation one, even though the post-operation one came within normal limits. In all cases the difference consisted of a higher vesical pressure on starting filling and a high pressure throughout filling.

Case 37.-This will serve as an illustration of the clinical picture found with division of the tracts unilaterally. The cystometrograms made a week before and a fortnight after the operation are shown as Figs. 11A and B; and the extent of the lesion made at operation is shown as Fig. 10. The cystometrogram (Fig. 11A) made before the operation is essentially normal; and the patient had no history to suggest any sort of abnormality of bladder functioning. Immediately after the operation, he had almost complete retention of urine; after an injection of carbaminoyl choline, he passed $120 \mathrm{ml}$., but a further $800 \mathrm{ml}$. remained in the bladder and had to be removed by catheter. For five days he was treated by tidal drainage. On the sixth day, he had a definite norma desire to micturate, he strained, and urine was passed beside the catheter. Tidal drainage was discontinued. Micturition from this time was apparently normal. The patient had a normal desire to micturate, he could feel urine passing and could feel the muscles of his perineum acting. He could micturate without straining, could start when he wanted to, and could stop in the middle of the act. The cystometrogram (Fig. 11B) made a fortnight after the operation is, however, far from normal. This is the typical cystometrogram of Stage II. On every occasion that the pressure rose, the patient had the desire to micturate, but felt it unilaterally. (This feature was described in our previous paper, 1951.) That he could control micturition is reflected in the rise of pressure to $20 \mathrm{~cm}$. when he was told to try and micturate. The comparison between the cystometrograms performed before and after the operation is striking. After the operation, the smooth slowly rising curve of the normal has gone, the base line, such as it is, is raised throughout, and spontaneous contraction occurs repeatedly, starting when there is less than $50 \mathrm{ml}$. of fluid in the bladder. $\mathbb{R}$ When this cystometrogram was made, the patient was unaware of having any disorder of bladder functioning, for he could control micturition normally. But the division of the pathway unilaterally has disturbed normal $\delta$ 을 bladder tone and has heightened the micturition reflex $\frac{1}{9}$ so that it is triggered off by inadequate stimulation.

Thus unilateral division of the centrifugal tract $\stackrel{ }{-}$ conveying impulses serving to inhibit bladder tone gives a change in vesical pressure - a change similar to but less than that which follows bilateral division of the tract. This might have been expected. For presumably less impulses arrive at the sacral centre from the centres in the brain, and so the total behaviour of the bladder resembles more nearly that of the bladder in cases of total cord division.

Cases of thrombosis of the posterior inferior cerebellar artery also show the syndrome of division of the tracts unilaterally. Although we have naturally not had the opportunity of performing cystometrograms before the thrombosis in such cases, cystometrograms performed after the thrombosis are similar to that given in Fig. 11B. The patient is usually unaware of any abnormality of bladder function, but the pressure is abnormally high, and the micturition reflex tends to occur frequently and with a relatively small amount of urine in the bladder. It is clear from such cases that in the medulla oblongata the fibres associated with the postural and reflex activities of the bladder must lie somewhere in the region between the inferior cerebellar peduncle and the olive, lateral 


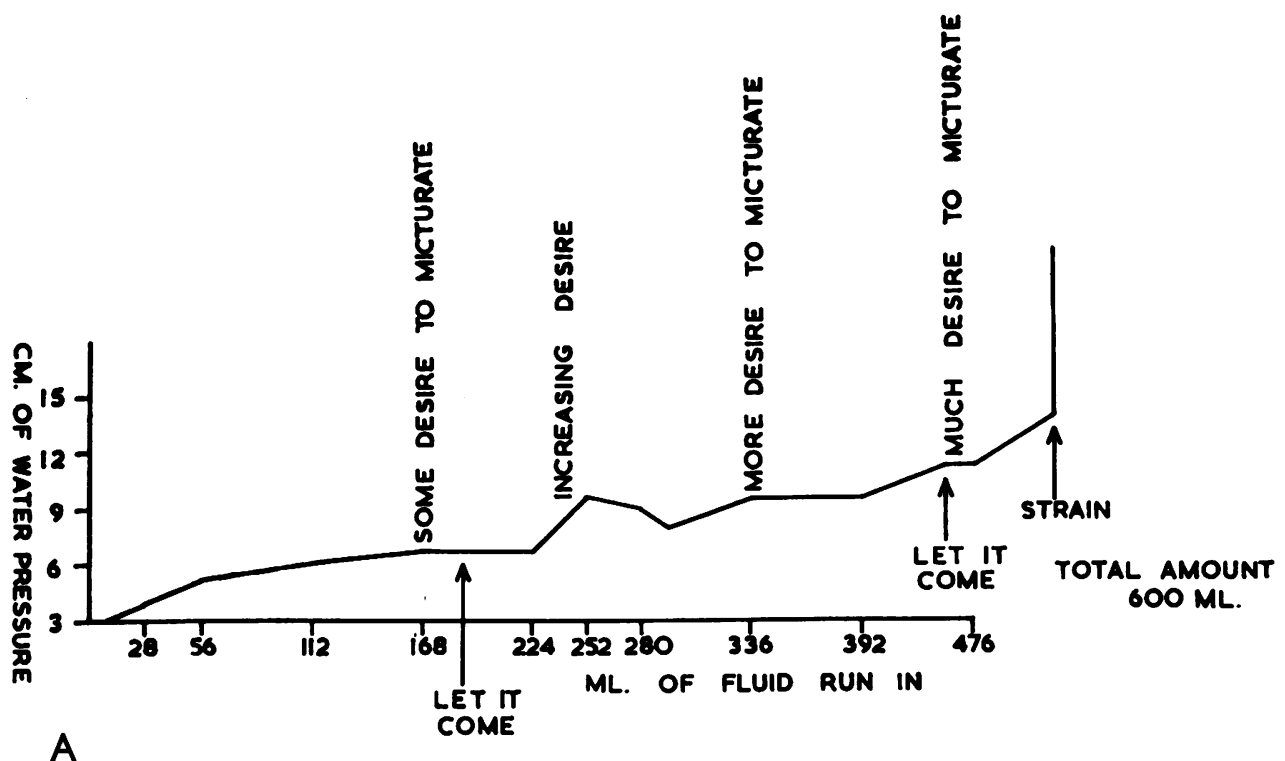

Fig. 11A and Fig. 11B.-Cystometrograms made one week before and two weeks after a unilateral cordotomy. The ability to control micturition on request is clearly seen to be present after the cordotomy: the pressure rose as soon as the patient was asked to let the urine come, and it fell again when he was requested to stop micturating. 
to the twelfth nerve. This is a region known to contain many fibres arising in the reticular substance, including reticulo-spinal fibres. At this level the more exact location of the fibres concerned with micturition cannot be worked out from our material. It may be concluded, however, that the fibres concerned do not form a part of the cortico-spinal tract.

\section{Conclusions}

The following conclusions may be drawn from our evidence. In man, the majority of descending fibres concerned with micturition lie in the lateral column, on an equatorial plane passing through the central canal. This location remains the same in the cervical, thoracic, lumbar, and sacral segments. These are the fibres mediating conscious control of the perineal and pelvic musculature, the fibres necessary for the starting and stopping of micturition, those concerned in inhibiting micturition, and those that inhibit the independent activity of the sacral bladder centre. It may be concluded that these fibres do not run in the median longitudinal bundle, the tecto-spinal tract, nor the vestibulospinal tract, for these tracts do not lie in the equatorial plane.

The centrifugal fibres run in close association with the centripetal fibres from the bladder subserving the sensation underlying the desire to micturate, and with the fibres subserving defaecation. We have previously shown (Nathan and Smith, $1951,1953)$ that the afferent fibres from the bladder and rectum lie in the lateral part of the lateral column, approximately on the plane of the central canal. The centrifugal fibres concerned with micturition are now shown to lie in the same plane, in a slightly more medial position; in the sacral segments, however, some of the efferent fibres lie on the periphery of the cord. We have been unable to separate the fibres concerned with conscious control from those concerned with the automatic, unconscious aspects of micturition. We have found some indication of a separation of the spinal fibres subserving defaecation from those subserving micturition. But the nature of our material-where the lesions made by the surgeon have to be large, and in which many of the patients have carcinoma in the pelvis-does not allow us to draw satisfactory conclusions about any differences in location of the fibres subserving these two functions.

\section{Discussion}

It has been shown that in man a bilateral operation on the spinal cord which divides the region on an equatorial plane passing through the central canal has an effect upon micturition similar to that pro- duced by a complete transection. Using the same technique, Barrington $(1933,1948)$ found the tracts run in the same region in the cat. Czyhlarz and Marburg's (1900) suggestion that these descending fibres run in man in the neighbourhood of the cortico-spinal tract is supported by our evidence, but their belief that " in order to get permanent disorder of bladder function " both cortico-spinal tracts have to be damaged to a large extent was not justified by our experience. Langworthy and Lewis's (1935) conclusion from two cases of disseminated sclerosis without post-mortem evidence that these tracts probably run in the lateral columns of the spinal cord in the neighbourhood of the cortico-spinal tracts is shown to be correct.

We observed in some patients more evidence of recovery than occurs after total division of the cord. Foerster and Gagel (1932) and Foerster (1936) state that they found this also. They concluded that there must be other tracts in the cord subserving micturition which had not been divided by the bilateral cordotomy incisions, and they thought that such tracts must run elsewhere than in the anterior or the antero-lateral columns. The argument, it would seem, is not so simple. To show that other efferent fibres are functioning, it is necessary to show that $\frac{\rho}{\circ}$ in all cases micturition recovers more than it would after complete section of the cord. However, even with total cord transection, bladder functioning eventually appears to be fairly efficient. And furthermore, somewhat better functioning after division of only the lateral columns is to be expected than after? total cord transection, for it is likely that other mechanisms are at the disposal of these patients, analogous to the trick movements used by patients with total divisions of peripheral nerves. For instance, they may have adequate information from the urethra to tell them that micturition is starting, progressing, and stopping, and they can take steps accordingly. In fact, experience has shown us that such patients sometimes have the impression that micturition is normal, when in fact all the spinal cord pathways are divided. They may notice only some degree of urgency. To the questions, Do you know when you need to pass urine, can you start micturition when you want to, can you stop passing urine in the middle of the act, the answer in each case can be, yes. And yet the patient may know when he needs to pass urine by paying attention to one of the substitute sensations, having lost the normal sensation underlying the desire to micturate; he may be able to start passing urine by means of increasing the intra-abdominal pressure or flexing forward while pressing his hands on his lower abdomen, having lost the normal mechanism; and he may be able to stop passing urine in the middle 
of the act, as he may have that kind of micturition which needs straining to be kept up throughout the act; if he ceases to strain, he ceases to pass urine; but he has lost the normal active contraction of the perineal muscles and sphincters that is needed to interrupt micturition in the normal way. If in any patient there is definite evidence that the recovery of bladder function has gone beyond what is possible with total division of the pathways subserving micturition in addition to the aid provided by an otherwise intact nervous system, then one is justified in believing that some pathways are intact. It is more reasonable to suppose that the fibres which have not been divided lie immediately adjacent to the area of the lesion made at operation than to conclude that they do not lie in the anterior or the antero-lateral columns as Foerster did. The reason for saying this is that all the patients we have seen went through the typical stages of bladder function that occur following complete division; if some then recovered to a more normal condition, it would seem that originally all the fibres were affected, but that some on the periphery of the lesion were only temporarily put out of action.

It might be objected to our argument that as in almost all our cases the centripetal pathway in the cord was divided at the same time as the centrifugal pathways, it is wrong to attribute all the effects we have described to the division of the centrifugal pathways, for they might be due to an inadequacy of information on the state of the bladder being received at the centres cranial to the spinal cord. But this objection seems to us to be invalid for three reasons. The behaviour of the bladder following division of these tracts is identical with that of somatic musculature following division of the relevant descending tracts. Both show a period of spinal shock, followed by a period of reflex hyperactivity and spasticity, and both show paralysis or severe paresis of consciously controlled movements. It is known that these effects on the somatic musculature result from the removal of the influence of the descending tracts. Further, the fact that in those cases in which the relevant tracts were cut unilaterally there was an increase in the tone of the bladder musculature would tend to implicate the descending tracts as responsible for these changes rather than the centripetal tracts; for it seems very likely that one afferent pathway intact on one side of the cord would suffice to convey information from the bladder to cerebral centres adequate for the normal coordination and control of micturition. Finally,
Case 11, of which the cord is shown in transverse section as Fig. 9, is important from this point of view; for this patient never regained the normal desire to micturate, the afferent pathways being divided, yet the tone and the reflex activities of the bladder returned to normal; and so here we had a case showing division of the spinal afferent pathways with normal bladder posture and coordination.

\section{Summary}

The location within the human spinal cord of the tracts subserving conscious control of micturition and the tracts subserving coordination of bladder functioning is determined.

These tracts are within the lateral column throughout the length of the spinal cord. Their approximate location is on an equatorial plane reaching from one periphery of the cord to the other, and passing through the central canal.

We wish especially to thank Mr. Wylie McKissock for his wholehearted cooperation. We are also grateful to the other neurosurgeons who have helped us and particularly to Mr. J. O'Connell. We would also like to thank Dr. E. A. Carmichael who gave us encouragement in this work and provided the facilities for it. To our technical assistants, Miss Anne Ebborn and $\mathrm{Mr}$. K. L. Frampton, we would like to express our gratitude for their careful and painstaking work.

\section{REFERENCES}

Barrington, F. J. F. (1933). Brain, 56, 126.

(1948). In Winsbury-White, H. P. Textbook of Genito-urinary Surgery. Livingstone, Edinburgh.

Budge, J. (1864). Z. rat. Med., 21, 1.

Czyhlarz, E. von, and Marburg, O. (1900). Wien. klin. Rdsch., $14,933$.

Dennig, H. (1926). Innervation der Blase. Berlin.

Denny-Brown, D., and Robertson, E. G. (1933). Brain, 56, 149 and 397

Foerster, O. (1936). In Bumke, O., and Foerster, O. Handbuch der Neurologie. Berlin.

-, and Gagel, O. (1932). Z, ges. Neurol. Psychiat., 138, 1.

Hunsicker, W. C., and Spiegel, E. A. (1933). Proc. Soc. exp. Biol. $(N . Y), 31,974$.

Langworthy, O. R. (1940). Res. Publ. Ass. Res. nerv. ment. Dis., 20,617

-, Kolb, L. C., and Lewis, L. G. (1940). Physiology of Micturition. Williams \& Wilkins, Baltimore.

Le, and Lewis, L. G. (1935). Bull. Johns Hopk. Univ., 56, 211. , P. M., and Langworthy, O. R. (1937). Amer. J. Physiol., 118,483

Mosso, A., and Pellacani, P. (1882). Arch. ital. Biol., 1, 291.

Müller, L. R. (1918). Dtsch. Arch. klin. Med., 128, 81

Nathan, P. W. (1952). J. Neurol. Neurosurg. Psychiat., 15, 148.

- (1956a). Ibid., 19, 101

(1956b). Brit. J. Urol., 28, 126

and Smith, M. C. (1951). J. Neurol. Neurosurg. Psychiat. $14,262$.

Nesbit, R (1953). Ibid., 16, 245

Nesbit, R. M., and Lapides, J. (1948). J. Urol. (Baltimore), 59, 726.

Sherrington, C. S. (1915). Brain, 38, 191.

Spiegel, E. A., and MacPherson, D. J. (1925). Pfügers Arch. ges. Physiol., 208, 570.

Stewart, C. C. (1899). Amer. J. Physiol., 2, 182.

Tower, S. S. (1940). Brain, 63, 36.

Wang, S. C., and Ranson, S. W. (1939). J. comp. Neurol., 71, 457.

Wruck, J. (1943). Z. ges. Neurol. Psychiat., 176, 179. 